\title{
Plasmonic properties of gold nanoparticles covered by silicon suboxide thin film
}

\author{
Evgeniy Baranov 1,*, Alexandr Zamchiy ${ }^{1}$, Aleksey Safonov ${ }^{1}$, Sergey Starinskiy ${ }^{1}$, Sergey \\ $\mathrm{Khmel}^{1}$ \\ ${ }^{1}$ Kutateladze Institute of Thermophysics SB RAS, 630090 Novosibirsk, Russia
}

\begin{abstract}
The optical properties of nanocomposite material consisting of gold nanoparticles without/with silicon suboxide thin film were obtained. The gold film was deposited by thermal vacuum evaporation and then it was annealed in a vacuum chamber to form gold nanoparticles. The silicon suboxide thin films were deposited by the gas-jet electron beam plasma chemical vapor deposition method. The intensity of the localized surface plasmon resonance increased and the plasmon maximum peak shifted from $520 \mathrm{~nm}$ to $537 \mathrm{~nm}$.
\end{abstract}

\section{Introduction}

In recent years, the light absorption enhancement in solar cells becomes the actual problem. One of the possible routes to solve this problem is using plasmonic nanoparticles for scattering of the light inside thin-film solar cell. Gold is a widely used metal with plasmonic properties. Localized surface plasmons of gold nanoparticles provide the light scattering at large angles without increasing the surface area of the solar cell. In addition, the using of plasmonic particles shifts the absorption maximum of the long-wave region of the spectrum [1]. It is possible to control the position of the absorption maximum by varying the parameters of the nanoparticles, such as size shape, concentration, as well as by changing the dielectric properties of the material around the particles.

Pulsed laser ablation method is simple and widely used technique that allows to obtain the gold nanoparticles in a wide range of sizes and concentrations [2]. The advantage of this method is that the material composition of the target fully reflects the composition of the synthesized nanoparticles.

In this paper, amorphous silicon suboxide $\left(\mathrm{a}-\mathrm{SiO}_{\mathrm{x}}: \mathrm{H}\right)$ thin films were used as a protective coatings. In addition, the changing of the stoichiometric coefficient of the $\mathrm{a}-\mathrm{SiO}_{\mathrm{x}}: \mathrm{H}$ thin films allows to control the plasmon resonance peak position. The aim of the work was to show that gold nanoparticles in a matrix of the a-SiO$: \mathrm{H}$ increase the intensity of its local plasmon resonance and the absorption maximum is shifted to long-wave region.

\section{Experiment}

\footnotetext{
* Corresponding author: itpbaranov@gmail.com
} 
The substrates were a silicon wafer (for measurement of particles size by a scanning electron microscope) and a borosilicate glass (for measuring transmittance spectra).

\subsection{Synthesis of the gold nanoparticles}

The gold films of about $4.8 \mathrm{~nm}$ thick were deposited by thermal vacuum evaporation onto the monocrystalline silicon and the borosilicate glass substrates. The pressure was $7 * 10^{-5} \mathrm{~Pa}$ during the deposition. The substrate temperature was $100^{\circ} \mathrm{C}$. Figure 1 shows the SEM image of as-deposited gold film with thickness about $4.8 \mathrm{~nm}$. It is evident from Fig. 1 that the film is not continuous and consists of individual particles of irregular shape with average size about $10 \mathrm{~nm}$.

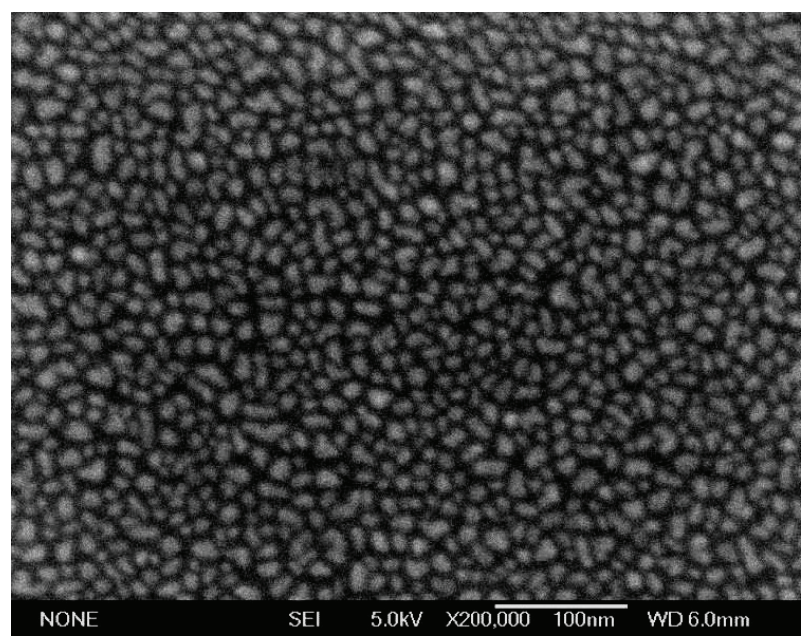

Fig. 1. SEM image of as-deposited gold film with thickness about $4.8 \mathrm{~nm}$.

Then the samples were annealed in a vacuum chamber at a temperature of $400^{\circ} \mathrm{C}$ for 1 hour to form gold nanoparticles [3]. Fig. 2a shows the SEM image of the gold nanoparticles on the silicon substrate obtained by a scanning electron microscope JEOL JSM-6700F. The Fig. $2 b$ shows a histogram of the size distribution of nanoparticles. The particles have a nearly spherical shape, average size is $13 \mathrm{~nm}$ and the filling density is low.

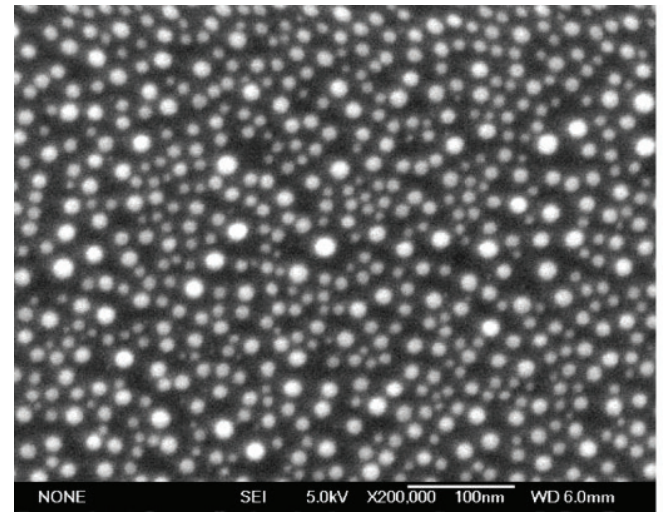

(a)

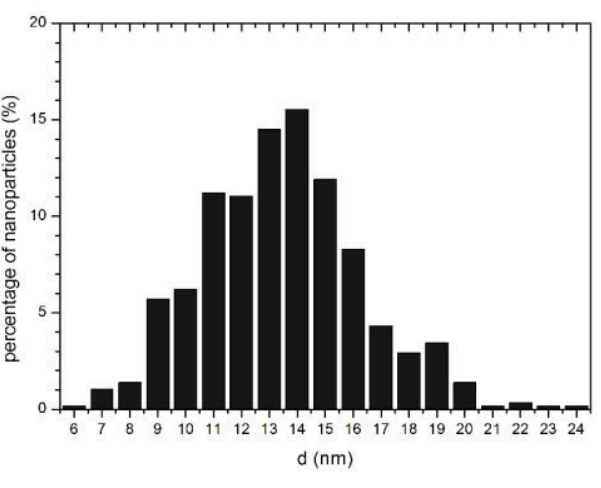

(b)

Fig. 2. a - SEM image of the gold nanoparticles on silicon substrate after annealing; b nanoparticles size distribution histogram obtained from SEM picture analysis. 


\subsection{Silicon suboxide thin films deposition}

In the present work, $\mathrm{a}-\mathrm{SiO}_{\mathrm{x}}: \mathrm{H}$ thin films were deposited by the gas-jet electron beam plasma chemical vapor deposition method [4]. Also, this method is used for the deposition of thin films of hydrogenated amorphous silicon [5] and the synthesis of silicon oxide nanowires $[6,7]$. The experiments were carried out in a vacuum chamber evacuated with a forevacuum pump. Gas pressure was not exceeding $16 \mathrm{~Pa}$ after introduction of processing gases (argon, a mixture of 5\% monosilane-argon, and oxygen). The gas flow rate was 280 $\mathrm{sccm}$ for argon $18 \mathrm{sccm}$ for the monosilane-argon mixture, and $6 \mathrm{sccm}$ for oxygen. Argon and the mixture were supplied into the chamber via a nozzle $2 \mathrm{~mm}$ in diameter, and oxygen was introduced directly into the vacuum chamber via a leak valve. For reagents activation, electron beam with an energy of $1600 \mathrm{eV}$ and a current of $70 \mathrm{~mA}$ was used generated by electron gun with a plasma cathode [8]. The substrates for films synthesis were c-Si $<100>$ wafers and Corning Eagle XG glass for infrared and optical measurements, respectively. The deposition time was 2 min that provided the samples thickness of about $130 \mathrm{~nm}$. Films were synthesized at substrate temperatures of $335^{\circ} \mathrm{C}$. Optical properties of silicon suboxide determined by using the software package PUMA.

\section{Results and discussion}

Figure 1 shows the extinction spectra of the gold nanoparticles without/with silicon suboxide thin film on the borosilicate glass obtained from transmission spectra. Oxygen concentration in the silicon suboxide film founded by FTIR measurement was $26 \%$ and hydrogen concentration was about $1 \%$.

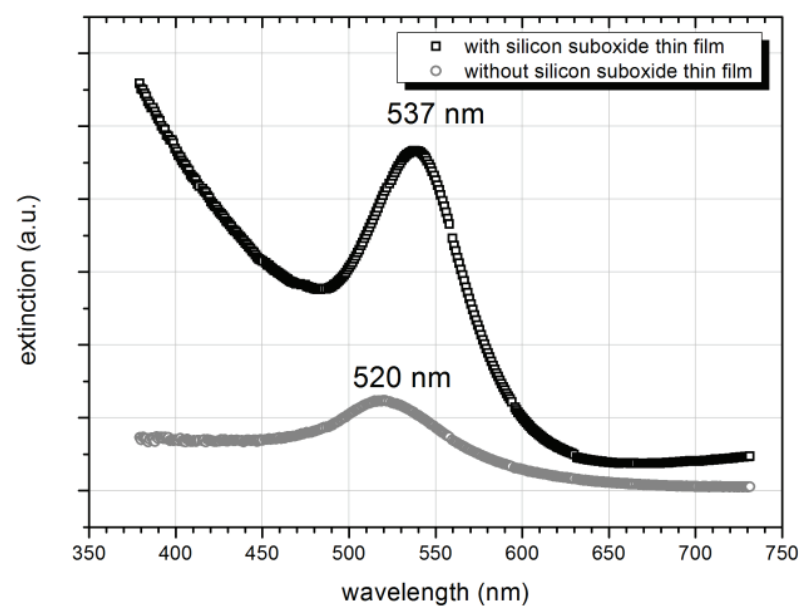

Fig. 3. Extinction spectra of the gold nanoparticles on the borosilicate glass: $\bigcirc$ - without silicon suboxide thin film; $\square-$ with silicon suboxide thin film.

Localized surface plasmon resonance of the gold nanoparticles on the borosilicate glass is observed at a wavelength of $520 \mathrm{~nm}$. This is characteristic of spherical nanoparticles, as shown previously in the literature [9]. When the gold nanoparticles were covered by the silicon suboxide film, plasmon peak maximum shifts to red wavelengths area. This is due to the refractive index change in the surrounding environment. The increase on absorption for wavelengths below $480 \mathrm{~nm}$ is due to the optical band gap of the material.

\section{Conclusion}


The gold nanoparticles with an average size of $13 \mathrm{~nm}$ were covered by silicon suboxide film with $130 \mathrm{~nm}$ thickness. In the issue, the intensity of the localized surface plasmon resonance increased and the plasmon maximum peak shifted from $520 \mathrm{~nm}$ to $537 \mathrm{~nm}$.

Research is supported by the Russian Science Foundation grant №16-10-10506.

\section{References}

1. E. Moulin, P. Luo, B. Pieters, J. Sukmanowski, J. Kirchhoff, W. Reetz, T. Müller, R. Carius, F.X. Royer, H. Stiebig, Appl. Phys. Lett. 95, 033505 (2009)

2. S.V. Starinskiy, Yu.G. Shukhov, A.V. Bulgakov, Technical Physics Letters 42, 411 (2016)

3. A. Safonov, V. Sulyaeva, N. Timoshenko, S. Starinskiy, Thin Solid Films 603, 313 (2016)

4. E. Baranov, S. Khmel, A. Zamchiy, M. Buyko, Physica Status Solidi (A) Applications and Materials Science 213, 1783 (2016)

5. E. Baranov, S. Khmel, A. Zamchiy, I. Cheskovskaya, M. Sharafutdinov, Can. J. Phys. 92, 723 (2014)

6. S. Khmel, E. Baranov, A. Zamchiy, E. Maximovskiy, D. Gulyaev, K. Zhuravlev, Physica Status Solidi (A) Applications and Materials Science 213, 1790 (2016)

7. S. Khmel, E. Baranov, A. Zaikovskii, A. Zamchiy, E. Maximovskiy, D. Gulyaev, K. Zhuravlev, Physica Status Solidi (A) Applications and Materials Science 213, 1774 (2016)

8. E. Baranov, S. Khmel, A. Zamchiy, IEEE Trans. Plasma Sci. 42, 2794 (2014)

9. A. Safonov, V. Sulyaeva, N. Timoshenko, K. Kubrak, S. Starinskiy, Physics Letters, Section A: General, Atomic and Solid State Physics 380, 3919 (2016) 